\title{
Model based determination of the influence of textile fabric on bioassay analysis and the effectiveness of a textile slow-release system of DEET in mosquito control Running Title: Influence of textile fabric
}

Benny Malengier ${ }^{\mathrm{a}}$, Tineke Goessens ${ }^{\mathrm{b}}$, Flora F. Mafo ${ }^{\mathrm{c}, \mathrm{d}}$, Mike De Vrieze ${ }^{\mathrm{e}}$, Lieva Van Langenhove ${ }^{\mathrm{a}}$, Samuel Wanji ${ }^{\mathrm{d}}$ and Frederic Lynen ${ }^{\mathrm{e}}$

\footnotetext{
${ }^{a}$ Department of Textiles, Faculty of Engineering and Architecture, Ghent University, Ghent, Belgium, tel. +32926457 35, fax +32926458 46, mail: benny.malengier@ugent.be

${ }^{\mathrm{b}}$ Research Group for Numerical Analysis and Mathematical Modelling, Department of Mathematical Analysis, Faculty of Engineering and Architecture, Ghent University, Ghent, Belgium

${ }^{\mathrm{c}}$ University of Yaoundé I, Yaoundé, Cameroon.

${ }^{\mathrm{d}}$ Research Foundation for Tropical Diseases and Environment, University of Buea, Buea, Cameroon.

${ }^{\mathrm{e}}$ Ghent University, Department of Organic Chemistry, Faculty of Science, Ghent University, Ghent, Belgium
}

\begin{abstract}
BACKGROUND: Determining how effective a product is to repel mosquitoes or other flying insects is a difficult task. One approach is using a bioassay with textile fabric. We investigated the role of the textile substrate in the bioassay with a numerical model, and compared with known results for DEET. We next apply the model to determine the effectiveness of textile slow-release formulations based on coatings, and compare this with a field study in the Cameroon.

Slow-release formulations are difficult to evaluate with standard tests as the compound needs a build up time not present in these tests.
\end{abstract}

RESULTS: We found excellent correspondence between the model and the known DEET results without matching parameters. Slow release approaches are deemed possible but have several drawbacks. Modeling can help in identifying optimal use conditions. The field test with a slow release system performed better than the model anticipated with initially more than $90 \%$ repellency. DEET coated textile was considered not marketable however.

CONCLUSION: We advise that bioassays also characterize more detailed the type of textile fabric used so as to allow drawing conclusions with textile modeling. Concerning coated textile slow release systems, more research is needed. We nevertheless advise usage mainly at entrance points, eg as scrims.

Keywords: spatial repellent, textile modeling, slow release, Anopheles, DEET 


\section{Introduction}

Mosquitoes transmit several pathogens that cause serious illnesses: malaria, yellow fever, dengue, and many more diseases. As a consequence, much research is done to determine new insecticides and mosquito repellents. Our work focuses on repellents, which have the ability to disrupt the host seeking abilities of the mosquitoes and induce movement away from the repellent. Once a product is determined to be a repellent, the next step is determining the effective dosage needed to optimally repel the mosquitoes, and how this dosage can be best provided.

Different testing setups exist to determine the spatial repellency of products. A common test is the arm-in-cage, in which a product is applied to an open section of a human arm, and introduced in a cage with up to 500 mosquitoes ${ }^{1}$. The difficulty with this test is the variability in attractiveness of the humans used, and the personnel cost. However, as the final aim of any spatial repellent is to repel mosquitoes from humans, an in vivo test like the arm in cage is a necessity.

Alternative in vitro tests have been developed. For example, cup designs like the Klun \& Debboun $(K \& D)$ test module $e^{2,3}$. In these a bait is used that mimics the human. Over this bait there is a cup, and mosquitoes are released in this cup. A difference here must be made between feeding deterrent, that is, a chemical that inhibits feeding when present in a place where the insects feed in its absence ${ }^{4}$, and spatial repellent, which is a chemical that causes mosquitoes to make a movement away from its source, and attraction inhibitors, which mask the presence of an attractant and hence prevent mosquitoes taking flight in the presence of an attractant and causing them to have difficulty locating the attractant. In cup tests the mosquito typically has no possibility to leave the cup, so the compound is tested like a feeding deterrent or attraction inhibitor. However, results are often used in the sense of a spatial repellent.

Depending on the tests, different textiles are used, or no textile at all. For example, nylon organdy cloths are used in some biting bioassays ${ }^{3,5}$, while others use muslin $\operatorname{cloth}^{5}$ for the repellency bioassay. The nylon cloth typically only serves as a delivery mechanism on top of the human skin or bait. The compound normally will remain as a layer on the nylon fibers, applied in such a way to not touch the human skin, and allowing mosquitoes to still bite the arm or bait underneath. Muslin cloth on the other hand consists of cotton fibers which will absorb the compound. This offers the possibility to test different concentrations of a compound.

In this study, we investigate with a model the influence of the textile used. In essence, for a repellent, the important aspect is the concentration in the air, which is almost never determined. 
Instead, different compounds and concentrations are applied to textile, which is the delivery system of the compound into the air. With a model this delivery system can be investigated. Several problems can occur with the textile which are of importance: the compound could bind with the textile aggregate causing an incorrect negative result, different research groups can use different textiles making the results not comparable, and different ventilation setups might be used resulting in very different air concentrations. With a model, the gradient of the compound in a domain (a cup or a room) can be determined also. This can help in determining if a feeding deterrent test might be valid as a repellent result: if a strong gradient is present in the environment of the mosquito, it stands to reason that the mosquito can move towards the lowest concentration, even if it cannot actually move away.

We will concentrate on DEET (N,N-diethyl-3-methylbenzamide) for this study, due to the large amount of information available on this successful insect repellent. DEET is an attraction inhibitor $^{6,7}$, which requires a bait to function as a repellent. Commercial products based on DEET obtain a mean time of protection of around 4 hours in academic studies ${ }^{1}$. Based on the known data, we can simulate the release of DEET, and the hypothetical result on mosquitoes.

Based on the result, tests were performed to create a slow release system for DEET based on the technology of the Belgian company Utexbel, enabling an extension of the protection offered by DEET. We evaluate the binding capacity of this system, it's theoretical performance over time, and the result of a field test.

\section{Simulation}

\subsection{Simulation Code}

Details about the simulation code can be found in an earlier article ${ }^{8}$. The code is freely available at https://gitorious.org/stickproject. The components of the code are:

1. A fiber model which implements a fiber as a layered cylindrical structure, with an evaporation law at the boundary.

2. A fiber-yarn multiscale coupling via an overlap zone in the fiber and yarn model. This allows to convert the system of a yarn consisting of hundreds of fibers into a single yarn model and 4 to 10 representative fiber models, interacting via a designated overlap zone. The fiber result in the overlap zone (corresponding to void space in the yarn model) is 
upscaled to the yarn overlap zone via a source/sink term, while the yarn result in the yarn overlap zone is likewise downscaled to the fiber model in the next time step.

3. A yarn model which implements the yarn as a cylindrical structure consisting of radial zones with a specific fiber composition. Every zone interacts with its own representative fiber model.

4. A yarn-fabric/environment multiscale coupling via an overlap zone in the yarn and fabric/environment models. This allows to reduce the problem of a fabric consisting of thousands of yarn threads into a fabric model interacting with some representative yarn models via a designated overlap zone. Now, the yarn overlap zone (corresponding to volume outside of the yarn) is upscaled to the fabric, and the fabric overlap zone (corresponding to the volume closest to the fabric) downscaled to the yarn in the next time step.

5. A fabric-environment model with basic ventilation. All test setups considered have a simple geometry allowing reduction to a $1 \mathrm{D}$ model.

The simulation code allows many different textile optimizations: changes in fibers, different blend proportions, weaving changes, etc.

Important in the model is how evaporation is handled. The evaporative flux $F_{\text {evap }}$ from skin is given as ${ }^{9}$

$$
F_{\text {evap }}(t)=\tilde{k}_{\text {evap }} \frac{\rho}{C_{m, \text { sat }}} C_{B}(t)
$$

where $k_{\text {evap }}$ is the evaporation coefficient, $C_{m \text {,sat }}$ the saturation concentration in the medium (eg skin), $\rho$ the density of the component, and $C_{B}(t)$ the concentration at the boundary. For a pure DEET layer, $C_{\mathrm{sat}}=C_{B}(t)$ and the equation reduces to

$$
F_{\text {evap }}(t)=\tilde{k}_{\text {evap }} \rho \text {. }
$$

One can interpret the $\frac{\rho}{C_{m, \text { sat }}}$ factor as a membrane partition coefficient. If effectively works like a porosity of the medium considered.

Equation (1) can be improved. We do this by considering a water liquid-vapor term ${ }^{10,11}$ : 


$$
F_{\text {evap }}(t)=S h_{l g}\left(C_{a, s a t}-C_{a, B}\right) H\left(C_{B}, C_{a, s a t}-C_{a, B}\right),
$$

where $S$ is the effective area fraction, $h_{l g}$ the mass transfer coefficient from liquid to gas (unit $\mathrm{mm} / \mathrm{s}), C_{a, s a t}$ the saturated concentration in air, $C_{a, B}$ the concentration of the component in the air at the boundary, $C_{B}(t)$ again the concentration at the boundary, and $H$ a Heaviside type function defined as:

$$
H(v, c)=\left\{\begin{array}{lc}
1, & c \leq 0 \\
0, & c>0 ; v<0 \\
1, & c>0 ; v>0
\end{array}\right.
$$

which indicates condensation happens if the control $c$ is less than 0 , while otherwise evaporation is governed by the presence of component at boundary. If we assume $C_{a, B} \approx 0$, and that the entire surface is available for evaporation $(S=1)$, the equation reduces to

$$
F_{\text {evap }}(t)=h_{l g} C_{a, s a t},
$$

which allows to obtain the approximate relationship

$$
\tilde{k}_{\text {evap }}=h_{l g} C_{a, s a t} / \rho \text {. }
$$

We further have that $S$ is proportional to $\frac{C_{B}(t)}{C_{m, s a t}}$, which shows that the improved equation (3) is indeed analogous to (1).

\subsection{DEET}

We concentrate on DEET as a lot information is known about this compound. Important for mosquito repellency is the air concentration needed for DEET to behave like a repellent. DEET is used primarily by dermal application as an insect repellent against mosquitoes, ticks, fleas, leeches, and blackflies. DEET is available in $4 \%$ to almost $100 \%$ concentrations in insect repellent formulations, including solutions, lotions, creams, gels, aerosols, pump sprays, and impregnated towelettes, usually with an ethyl or isopropyl base.

In the atmosphere, it exists in the vapor phase and is degraded by reaction with photo-chemically produced hydroxy radicals; its atmospheric half-life is approximately 15 hours. DEET has moderate mobility and is not expected to volatilize in moist or dry soil or to biodegrade under either aerobic or anaerobic conditions. 
A minimum evaporation rate of $5 \mu \mathrm{g} /\left(\mathrm{cm}^{2} \mathrm{~h}\right)\left(0.03 \mu \mathrm{mol} /\left(\mathrm{cm}^{2} \mathrm{~h}\right)\right)$ for DEET over $5-15$ minutes was determined for human skin. DEET then acts as a volatile agent to repel mosquitoes at distances of at least $38 \mathrm{~cm}$ from their host ${ }^{12}$.

The question on how DEET repels mosquitoes has been investigated in different studies. One such study ${ }^{13}$ stated that DEET inhibits the 1-octen-3-ol-evoked responses by inhibiting the activity of the olfactory receptory neurons (ORN's) on the antennae of the mosquito. Another study ${ }^{14}$ provided convincing evidence suggesting that repellency of the mosquito is a matter of direct detection of DEET in the vapor phase and avoidance of the smell of DEET. The researchers found a DEET-sensitive ORN and pointed out a false positive in the previous research due to trapping of odorants in the Pasteur pipes when a DEET-laden filter paper was added to the cartridge. This means the mosquitoes couldn't smell DEET due to this fact and not due to inhibition of the olfactory system.

We estimate the diffusion coefficient of DEET in air according to the methods used by the Emission Standards Division ${ }^{15}$, which gives $D_{g, D E E T}=0.07778 \mathrm{~cm}^{2} / \mathrm{s}$.

From Santhanam et al (2005) we can estimate the evaporative flux of DEET around $7.210^{-3} \mu \mathrm{g} /\left(\mathrm{cm}^{2} \mathrm{~s}\right)$. This translates in a buildup of several microgram of DEET after some minutes. The different evaporative laws (1)-(3) have corresponding coefficients $\tilde{k}_{\text {evap }}=7.210^{-8}$ $\mathrm{mm} / \mathrm{s}$ and $h_{\mathrm{lg}}=0.897 \mathrm{~mm} / \mathrm{s}$.

Pure DEET evaporates faster than needed to repel mosquitoes, so a slow release system would be beneficial. It has been determined ${ }^{16}$ that a concentration of $2 \mu \mathrm{g} / 1$ air of DEET is needed for repellency. Karr et al used an encapsulation technique ${ }^{17}$ to slow down the release. They determined that to repel mosquitoes a minimum effective evaporation rate of $3.3 \pm 0.8$ $10^{-4} \mu \mathrm{g} /\left(\mathrm{cm}^{2} \mathrm{~s}\right)$ should be achieved. The different evaporative laws (1)-(3) then have corresponding coefficients $\tilde{k}_{\text {evap }}=3.310^{-9} \mathrm{~mm} / \mathrm{s}$ and $h_{\mathrm{lg}}=0.041 \mathrm{~mm} / \mathrm{s}$, indicating the evaporation can be reduced a factor 22 and still give rise to repellency.

\subsection{Muslin cloth}

The muslin cloth used in bioassays is normally not characterized. Therefore, we consider some typical values. We consider a fabric of 5 on $10 \mathrm{~cm}$, consisting of yarns which are $0.6 \mathrm{~mm}$ apart center to center in vertical and horizontal direction. A yarn has radius of $0.105 \mathrm{~mm}$ and typically contains 160 cotton fibers. A cotton fiber has a typical radius of $0.0052 \mathrm{~mm}$ and density of 1.55 
$\mathrm{g} / \mathrm{cm}^{3}$. The moisture regain (absorbed water expressed in percentages of the dry weight) of cotton at $65 \%$ relative humidity $(\mathrm{RH})$ at $20^{\circ} \mathrm{C}$ is 6 to $7 \%$, and $20 \%$ at $100 \% \mathrm{RH}$, indicating that cotton can absorb water up to $20 \%$ of it's own weight in water. If we assume the used muslin cloth to be originally fully dry, we can conclude that also for DEET $20 \%$ regain is possible, allowing a maximum of $0.31 \mathrm{~g} / \mathrm{cm}^{3}$ in the cotton, which translates to a porosity of DEET in cotton of $n=0.31$.

The total volume of cotton in the muslin cloth is $V_{c}=228.6 \mathrm{~mm}^{3}$, which leads to a maximum absorbed DEET content of $0.071 \mathrm{~g}$ or $1.4 \mathrm{mg} / \mathrm{cm}^{2}$.

In a typical bioassay a stock solution is diluted to produce test concentration of $1.5,0.75$, $0.375,0.187,0.094,0.047,0.023,0.011$ and $0.006 \mathrm{mg} / \mathrm{cm}^{2}$, which corresponds with a dose range of 7841 down to $31 \mathrm{nmol} / \mathrm{cm}^{2}$. We see that these concentrations can be absorbed by the muslin cloth, except for the first, which should result in a thin surface layer of not absorbed DEET. Converting this to the fraction $n_{D}$ of the porosity available used, we obtain $1.07,0.54,0.27,0.13$, $0.067,0.033,0.017,0.008,0.004$. The article we compare with uses also $25 \mathrm{nmol} / \mathrm{cm}^{2}$ or 0.0048 $\mathrm{mg} / \mathrm{cm}^{2}$, which translates to a used fraction $n_{D}$ of 0.0034 . These fractions allow us to correctly set initial conditions for the numerical model. We set in (3)

$$
S(t)=n \frac{C_{B}(t)}{\rho},
$$

and consider as initial DEET concentration in the fiber

$$
C(0)=C_{B}(0)=n_{D} \rho,
$$

with $n_{D}$ the fraction of the porosity used for the start concentration.

\subsection{Numerical Experiments}

As a first experiment, we compare with Fig. 4 of Ali et $\mathrm{al}^{5}$, which is a dose response study for DEET at $25,20,15,10,5 \mathrm{nmol} / \mathrm{cm}^{2}$. In that study, $25 \mathrm{nmol} / \mathrm{cm}^{2}$ provides repellency of $90 \%, 20$ and $15 \mathrm{nmol} / \mathrm{cm}^{2}$ gave equal reduced repellency of $70 \%$, while 10 and $5 \mathrm{nmol} / \mathrm{cm}^{2}$ showed already results similar to solvent control. In the dose response, the fabrics are dried for 3 to 5 minutes, which we estimate can maximally remove $1 \mu \mathrm{g}$ of DEET, which is sufficiently low. The patch is attached to a human arm, which is then introduced in a screened cage with mosquitoes exhibiting host seeking behavior. The movement of the arm will create an unknown amount of 
forced convection over the muslin cloth. The arm is kept 1 minute in the cage, after which it is counted how many mosquitoes could feed.

As we cannot know the influence of the forced convection, we simulate this with a run of the simulation code over 2 minutes, starting with the initial known concentration and no DEET present in the surrounding air. We then considering the last minute of the simulation as meaningful to compare with the result of Fig. 4 of Ali et $\mathrm{al}^{5}$. This because the forced convection will remove the surface layers above the muslin cloth containing DEET, but not the DEET concentration accumulated inside the cloth void spaces. The simulation results are shown in Fig. 1.

The results nicely match the bioassay: $25 \mathrm{nmol} / \mathrm{cm}^{2}$ reaches the required minimum amount of 2 $\mu \mathrm{g} / 1$ air of DEET almost for the entire duration of the last minute of simulation. The 10 and 5 $\mathrm{nmol} / \mathrm{cm}^{2}$ however remain under $2 \mu \mathrm{g} / 1$ air of DEET, and cannot offer protection against the mosquitoes.

We stress that no parameter matching was done, all parameters used in the simulation have been estimated based on previous DEET studies. Hence, this test could have been used to estimate the required amount of DEET in the air for repellency.

As a second experiment, we consider the case of the application of $25 \mathrm{nmol} / \mathrm{cm}^{2}$ on the muslin cloth and doing a duration test. These results should be compared with the results in Fig. 3 of Ali et $\mathrm{al}^{5}$. The result there can be summarized as the muslin cloth giving almost full protection for 2 hours, after which there is a linear decrease, with at 6 hours still a repellency of $70 \%$ (proportion not biting), while after 12 hours there is little difference with the positive control. We simulate this by considering a sample that evaporates in an open environment with initial concentration 25 $\mathrm{nmol} / \mathrm{cm}^{2}$. At $0,1,3,6,12$ and 24 hours, the sample is moved for testing to a cage. Hence, we consider the obtained concentrations at those time points, and do tests as in our first numerical experiment with those values.

We obtain the Fig. 2, of which the relevant data to compare with the reference is given in Table 1. Comparing with the results in Fig. 1, we see that a good correspondence with the experiment is obtained. In Fig. 3, we show the protection over a day given by the muslin cloth if the cloth would remain fixed in position. The bottom dashed line in Fig. 3 corresponds to the determined lower 
boundary of DEET concentration needed to have good repellency. By moving the muslin cloth to the testing cage, the surface layer above the fabric will be replaced by mostly fresh air (forced convection), and hence the protection right after movement (Fig. 1) is considerably decreased from this ideal consideration of an arm that remains fixed in position. By the movement of the user, the result after 6 hours is already much reduced due to the slower buildup of DEET at the lower concentrations, while not moving the muslin cloth gives good protection even at $5 \mathrm{~mm}$ for the full 24 hours. It is important to note that forced convection over the muslin cloth would change this considerably.

In the simulation, air diffusion and convection reduces the DEET concentration around the muslin cloth at a slow rate as the model mimics an open cup evaporation.

Although the computational results match the experiment satisfactory, not everything was taken into account. The following processes will lead to differences:

- The muslin cloth sample was soaked in a DEET solution in a closed cup, and allowed to dry for 3 to 5 minutes after extraction from the cup. The closed cup will see a high DEET concentration, lowering the actual DEET concentration present in the muslin cloth. We estimate this can reduce the total DEET mass by a maximum of $1 \mu \mathrm{g}$. As $5 \mathrm{nmol} / \mathrm{cm}^{2}$ corresponds with $30 \mu \mathrm{g}$, this is a small error lowering the actual effectiveness.

- We assume in the model that all DEET was absorbed in the cotton fibers. In reality, some will remain attached to the surface, where it can evaporate fast at the beginning. This also reduces the amount of DEET present when the experiment starts. As the saturation concentration for DEET can be easily obtained close to the fiber in both cases for the concentrations absorbed considered, we estimate this effect to be neglectable.

- The muslin cloth used in the original paper is not given, but can have some influence on the results. For the simulation we consider yarns which are $0.6 \mathrm{~mm}$ apart (midline to midline), with a yarn radius of $0.105 \mathrm{~mm}$, consisting of 160 cotton fibers of radius $5.2 \mu \mathrm{m}$, all values typical for cotton cloth. Tests with the simulation code show the effect of changes to be small within realistic variations.

- We consider a slow convection, driven by a boundary condition of 0 sufficiently far from the muslin cloth. Any actual forced convection, as can be expected from time to time in an 
experimental environment, will reduce the DEET concentration faster than the model considers. This can be assumed to have a large influence. We take it into account by setting the outside concentration to 0 when movement occurs (moving the hand in a test cage), and add a minute of simulation time to determine a new initial outside concentration.to work with.

- We give the concentration of DEET at 1 or $5 \mathrm{~mm}$ away from the target in Fig. 1 and 3 as values to determine if repellency is working or not. It could be that concentrations farther away from the muslin cloth must be considered for effective repellency (as the host attraction might overrule the DEET present at these distances). As DEET is considered to effectively repel the mosquitoes, considering the value at $1 \mathrm{~mm}$ should be a good assumption for the cage test, while for static conditions $5 \mathrm{~mm}$ seems a good sampling position.

We conclude that we obtain with these assumptions the same results as the actual bioassay using as only input the known characteristics of DEET, and that it seems that the minimum required concentration for repellency of a new product could be determined with the method, if it was not previously known.

\section{SLOW RELEASE}

\subsection{Lab Tests}

The results obtained show there is room to develop a slow release adaptation. Such an application might require some time to achieve repellency, but would not deplete as fast. Utexbel, a Belgian textile company, provided us with textile samples where DEET was embedded in a polymer coating. In this paper we do not concern ourselves with the actual polymer coating, which is an internal Utexbel product, but instead characterize it's effect. The coating process used was analogous to the one Utexbel has available for permethrin in their BuzzX (http://www.buzzx.info/) range of products. We wanted to investigate if a slow release system for DEET can be obtained like this, as permethrin has very low vapor pressure and is not considered volatile.

A different slow release systems based on encapsulation have been tested before ${ }^{17,18}$ with varying results. Depending on the encapsulation used the release of DEET can be spread over 1 week to several months. Encapsulation is an interesting technique, but obtaining the optimal 
release rate of repellent is not straightforward. It requires a step to create the capsules, and another step to bind the capsules to the fabric. A polymer coating technique has the advantage that it is a coating technique, comparable to other textile finishes. Which technique is preferred will depend on production considerations. The modeling presented can be adapted to simulate release by microcapsules provided a model is available for how fast the capsules release DEET.

The samples received from Utexbel had to be characterized in terms of DEET content. Doing mass balance experiments to determine evaporation rate proved troublesome as the polymer layer and textile will only slowly come to equilibrium when exposed to a different relative humidity. A test showed that in a climate chamber, several hours were needed to reach equilibrium, during which the sample increased in weight. As a weight test would need to run longer than we could reserve the climate chamber, a chemical approach was chosen instead using GC-MS. Like this also the actual initial amount of DEET can be obtained. Hence, the samples were tested on arrival, and after 91 days. In between, the samples were kept under a fume hood. GC-MS was used to determine the DEET and permethrin content. A HP6890 series GC system coupled to a HP5973 MS system was used, with an injection volume of $1 \mu \mathrm{L}$ for liquids. SPME stayed in the inlet for the whole run. The column used was HP- 5MS, $30 \mathrm{~m} \times 0.25 \mathrm{~mm}$ I.D., $0.25 \mu \mathrm{m} \mathrm{df}$, with as temperature program $40^{\circ} \mathrm{C}$ to $300^{\circ} \mathrm{C} @ 10^{\circ} \mathrm{C} / \mathrm{min}$. The liquid extractor analysis occurred on strips of 4 by $0.5 \mathrm{~cm}$ obtained from the samples. They were extracted with $3 \mathrm{~mL}$ methyl-tert-butyl-ether (MTBE) in a $4 \mathrm{~mL}$ vial. 30 minutes sonication provided $>85 \%$ recovery for the DEET and permethrin. Calibration was done with pure DEET and a stock solution of permethrin.

For permethrin, no reduction in concentration was found after 91 days, as was expected, as permethrin is not volatile. The results for DEET can be found in Table 2, where from the given measurements the long term evaporative flux $F_{\text {evap }}$ is estimated, and like this also long term values for $\tilde{k}_{\text {evap }}$ and $h_{\mathrm{lg}}$. Note that for this, the fiber surface is used, and not the muslin cloth surface of $2 \mathrm{~cm}^{2}$. To obtain this fiber surface, we took into account that the samples were a blended fabric with $65 \%$ cotton and $35 \%$ polyester. The cotton fiber has an elliptical shape with average long axes $0.0183 \mathrm{~mm}$ and short axis $0.01339 \mathrm{~mm}$. The polyester fiber has average radius $0.00551 \mathrm{~mm}$. There are 190 fibers in a yarn, and 27 yarns per $\mathrm{cm}$ in the vertical direction, and 43 yarns per $\mathrm{cm}$ in the horizontal direction. As a consequence, we estimate the fiber surface where evaporation occurs to be 29 times the fabric surface. 
We obtain that the polymer treatment reduced the release of DEET enormously, with $h_{\mathrm{lg}}$ dropping from $0.897 \mathrm{~mm} / \mathrm{s}$ in pure form to a value between $0.0610^{-4}$ and $0.310^{-4} \mathrm{~mm} / \mathrm{s}$, or a reduction of 29900 to 149500 . However, in the deduction, we have assumed the entire area of the fiber surface coating is available for evaporation, so $S=1$. Previously, for the $25 \mathrm{nmol} / \mathrm{cm}^{2}$ dilution on cotton, we had, see (6), $S=0.001$, so this cotton sample which had good repellent properties also had a reduction in evaporation compared to pure DEET of 1000 . We conclude that a further reduction of 29.9 to 149.5 in evaporation speed was obtained after polymer coating. In other words, where Fig. 2 shows that the $25 \mathrm{nmol} / \mathrm{cm}^{2}$ sample was almost depleted after 24 hours, for the polymer coated version with the same concentration, this would be around $24 \times 29.9$ hours, or after 30 days. Compared with the needed evaporation $\operatorname{rate}^{17}$ of $F_{\text {evap }}=3.3 \pm 0.810^{-} 6 \mu \mathrm{g} /\left(\mathrm{mm}^{2} \mathrm{~s}\right)$ the determined $\mathrm{F}_{\text {evap }}$ of our textile slow release system seems a factor 35 to 58 too low. Note however that the fiber surface of textile is typically 25-30 times larger than the textile surface, so this discrepancy can be overcome by selection of the fabric used to construct the repellent textiles.

The estimates done are rough, but nevertheless give an idea of the order of magnitudes to work with. We are specifically lacking in knowledge about the polymer: how does DEET diffuse in the polymer, how is the evaporation process working? We can deduce from Table 2 that evaporation of DEET will be higher at higher concentrations in the polymer. We have however too few data points to fully characterize the evaporation behavior. If we consider a least-squares fit through the points formed by the midpoint concentration $\left(\mathrm{C}_{\text {start }}+\mathrm{C}_{91 \text { days }}\right) / 2$, and the long term computed $h_{\mathrm{lg}}$ in Table 2 , we obtain

$$
h_{\lg }=10^{-7}(4.9+2 C)
$$

with $C$ the concentration on this textile in $\mathrm{nmol} / \mathrm{cm}^{2}$.

For the modeling we will use following assumptions:

- We assume the evaporation is not diffusion limited. That is, diffusion in the polymer is faster than evaporation, and no specific knowledge about the diffusion process is needed apart from the fact it is sufficiently fast

- By lack of knowledge about the specific binding of DEET in the polymer, we will set in (3) $S=1$, and use $h_{\lg }$ from (8).

- The derivation for (8) is for the tested textiles. Other textiles can use the same formula provided their fiber surface area is comparable. That is, the evaporation rate depends on the 
DEET concentration in the polymer layer, not on the measured fabric surface concentration expressed in $\mathrm{nmol} / \mathrm{cm}^{2}$.

As evaporation is now a slow release process, this modeling will be adequate to obtain qualitative results. Having $S=1$ and $h_{\mathrm{lg}}$ fixed based on (8) will allow to see the effectiveness of a textile over a typical night. We consider a bed net that has comparable total fiber surface area as the tested textiles, and consider an initial concentration of 400, 200, 100 and $50 \mathrm{nmol} / \mathrm{cm}^{2}$. This corresponds, with $S=1$, to mass transfer coefficient $h_{\lg }$ of $810^{-5}, 610^{-5}, 410^{-5}, 210^{-5}$, and $10^{-5}$. A reduction from 400 to $50 \mathrm{nmol} / \mathrm{cm}^{2}$ will occur over 3 to 4 months, or, if carefully stored in a plastic bag and only used for 8 hours on a day, over a year. We don't simulate values above $400 \mathrm{nmol} / \mathrm{cm}^{2}$ although that is theoretically possible to obtain. This because based on Table 2, we can't assume (8) is still valid at higher concentrations.

The results of the simulation are shown in Fig. 4. Here we see the effect of a sheet or curtain of the bed net textile put in the middle on a rectangular room. To interpret this Figure, we need to consider that an actual bed net will consist of a sheet to the right and left of the bed, so the concentrations observed will be at least double that of a single sheet. As we are far from the saturation concentration of DEET, we can indeed approximate the effect of the bed net by doubling the simulation result, which is indicated with dashed lines in Fig. 4.

The simulation uses a 1D representation of the room, so it is currently not possible to take the actual geometry of a testing hut (eg. window traps) into account. As we indicated, the buildup of DEET around the bed net is slow. Considering the double action of a net, we reach protection against mosquitoes at $1 \mathrm{~mm}$ after $70 \mathrm{~min}$ for the $400 \mathrm{nmol} / \mathrm{cm}^{2}$ bed net. Further away from the bed net, more time is needed. Forced convection would spread the results faster over the room, which would have a positive effect. The actual ventilation of the room would have to be taken into account to fully match the experimental setup. This could lead to higher or lower values depending if the ventilation is lower or higher than considered in the model. The model uses a fixed boundary condition of 0 DEET at $2.5 \mathrm{~m}$ to simulate the ventilation.

Qualitatively we can deduce from the simulation that

1. A slow release system does not give adequate protection for the first hour. In reality however, the product would be stored in a bag, which would create a buildup of DEET in the bag and in the void zones of the textile. As a consequence, in reality full protection 
might be present from the start, depending when and where the bag is opened, if the forced convection is sufficiently low.

2. Over time, provided sufficient initial concentration is present, the bed net will provide full protection from mosquitoes present in the room. It therefore offers effective protection from entry via cuts or holes in the bed net.

3. To avoid entrance of mosquitoes into the sleeping chamber, the slow release system needs still more time. At $200 \mathrm{nmol} / \mathrm{cm}^{2}$, even after 8 hours there is not sufficient DEET at the entry points to prevent entrance. As real rooms will have varying size, using a DEET treated bed net commercially to avoid mosquitoes in the room does not seem feasible.

As a consequence, following recommendation can be given:

1. To maximize the effective duration of the treated textiles, they would better be used only at the points of entrance of the mosquitoes, e.g. as a curtain or scrim (light gauzy material).

2. It is useful to add DEET to bed nets so as to avoid biting through holes in the bed net or due to body parts touching the bed net. The initial hours however need to be overcome to achieve full protection. This could be done in some other ways, e.g. by using a spray before going to bed.

3. In practical use, a consumer would need to be made well aware that a repellent textile has a limited durability, governed by the initially applied dose. Our modeled bed net with $400 \mathrm{nmol} / \mathrm{cm}^{2}$, corresponds to a use of only $76 \mu \mathrm{g} / \mathrm{cm}^{2}$ of DEET, which is a very modest use of repellent. If higher amounts can be added to a slow release coating, a long adequate extra protection could be offered as compared to current pyrethroid only bed nets.

\subsection{Field Tests}

Based on the knowledge learned, 2 types of bed nets (Utexbel type Y412 and Y335) were coated for field testing in the Cameroon. Field testing should realistically evaluate the efficacy of the slow release system, as the technique will require an accumulation of active component, making a short arm in cage test in a small cage not indicative. At the same time, a field test can be considered as the gold standard for testing.

A total of 5 different coatings were prepared: DEET with and without washing binder, DEET combined with permethrin with and without washing binder, and permethrin without washing binder. The washing binder should have no influence on the performance, it is needed for textile 
treated with permethrin to keep the permethrin stable and to avoid washing removing the polymer layer.

The DEET content was maximized as much as possible. As a consequence the bed nets had an oily feel. A typical DEET odor could also be distinguished. The nets would probably not be marketable for those reasons. As the fabrics used in GC-MS did not have this, we can conclude that the concentration is considerably higher than $400 \mathrm{nmol} / \mathrm{cm}^{2}$ obtained as maximum in those tests. Furthermore, the production process was manual. The upscaling of DEET application to an industrial finishing process involves some challenging issues like

- environmental specifications concerning the volatile concentration in the ambient air and preventing operators getting into contact with those vapours

- minimizing contamination of the process equipment

- managing cleaning procedures

- managing recycling issues

- dealing with the aggressivity of DEET towards plastic parts, including end user packaging.

Testing was done at the research institute REFOTDE, Cameroon. The textiles where cut and knitted into usable bed nets. Three experimental huts in Meanja were used. In this location, the testing huts are between a breeding place and a small community. The testing schedule was done based on a Latin Square design, with a positive control present during all tests in a fixed hut, and 1 net in the other huts used for 4 days. The nets were mounted each evening, remaining exposed for 12 hours (from 6pm to 6am), dismounted the next morning, kept in plastic bags, and mounted again the next evening (12 hours a day for 4 days).

A hut functionality test showed that $18.4 \%$ of mosquitoes were captured inside the huts. An average number of 4.5 mosquitoes/hut/night was found in this test. The dominant species was Anopheles (50\%), followed by Mansonia (48\%) and Aedes (1\%). A schematic of the sleeping hut is given in Fig. 5.

The results with the different nets is given in Table 3. In this, repellency is defined as the reduction in entry rate into a hut, while feeding inhibition is the reduction in feeding (so (\# control - \# test)/ \# control as percentage). In the control an untreated net is used, so also in the control it is possible to have no mosquitoes feeding over the 4 days of testing. 
From the Table, we see that the bed nets with DEET performed better than anticipated by the model: they could prevent mosquitoes from entering the hut (column \% Repel), in 2 cases even for $100 \%$. For the products with DEET, not a single fed mosquito was recorded. In the case of a traditional permethrin treated net, we can see that the repellency drops to 66-77\%, but the mosquitoes that entered were also prevented from feeding due to the permethrin coating. The DEET treated nets also cause all mosquitoes to be found in the veranda.

To validate that the products can survive storage, the nets were stored for 12 months in plastic bags, and the best performing retested. The results of this retesting are in Table 4. We note that one product had a seriously reduced functionality, with the repellency dropping to $66 \%$. The other 3 products however performed only slightly less good than in the original test.

\section{DISCUSSION AND CONCLUSION}

We can conclude from the results that a slow release system for DEET can be developed, and that the effect of DEET is recognizable in the field testing, giving the best possible protection. The slow release is optimized in such a way that no excessive amount of DEET is used, maximizing the time to depletion. We attribute the better values than obtained by the model to a higher initial concentration than the $400 \mathrm{nmol} / \mathrm{cm}^{2}$, and the preparation time before testing starts $(6 \mathrm{pm})$ which reduces the time needed for building up an initial concentration of DEET in the sleeping huts.

However, the sleepers complained that the DEET nets were sticky and had an oily feel. The odor was also considered unpleasant over the night. As indicated before, the production process can also not be upscaled. All this indicates that further research should be performed to bind a more pleasant repellent in a slow release product, or to adapt the production process with DEET in a way that is feasible for industrial production. Like this the high repellency (mosquitoes not entering the sleeping huts) values found when using DEET bet nets can become a reality for people around the world 
We have modeled repellent compounds being released from textile fabrics. Comparison with known results for DEET bioassays shows that the observed data, be it active working duration of DEET or dose response study, can be explained via the study. This opens the possibility to determine effective air concentration needed of repellents via a coupling of the numerical model and a bioassay. Next, a slow release model has been developed for repellents applied to textile. In this one can adapt the textile properties, specifically the effective fiber surface, in such a way as to optimally interact with a reduced evaporation due to polymer coating. A test was done with bed nets in the Cameroon showing that this approach is viable. Further research should be done however to repeat this approach with a more pleasant repellent, or to convert the coating technique of DEET to allow higher dosage in the textile fabric so as to achieve a longer durability. The modeling should also be extended so as to allow a correct representation of the room used and a correct inclusion of the effect of air movement on the repellent concentration in and around the textile. Based on the model of a slow release product we would nevertheless advise to consider slow release products mainly at the entry points, for example in the form of a scrim.

\section{ACKNOWLEDGEMENTS}

We wish to acknowledge the support from European Union project: NMP2-2009-228639, FP7, NOBUG project.

\section{REFERENCES}

1. Masetti A, Maini S. Arm in cage tests to compare skin repellents against bites of Aedes albopictus. Bulletin of Insectology. 2006;59(2):157-160.

2. Klun JA, Debboun M. A new module for quantitative evaluation of repellent efficacy using human subjects. J Med Entomol. 2000;37:177-181.

3. Klun JA, Kramer M, Debboun M. A new in vitro bioassay system for discovery of novel human-use mosquito repellents. Journal of the American Mosquito Control Association. 2005;21:64-70.

4. Dethier VG, Browne BL, Smith CN. The designation of chemicals in terms of the responsesthey elicit from insects. J Econ Entomol. 1960;53:134-136.

5. Ali A, Cantrell CL, Bernier UR, Duke SO, Schneider JC, Agramonte NM, et al. Aedes aegypti (Diptera: Culicidae) Biting Deterrence: Structure-Activity Relationship of Saturated and Unsaturated Fatty Acids. J Med Entomol. 2012;49(6):1370-1378.

6. Davis EE, Bowen MF. Sensory physiology basis for attraction in mosquitoes. J Am Mosq Control Assoc. 1994;10:316-325.

7. Dogan EB, Ayres JW, Rossignol PA. Behavioral mode of action of DEET: inhibition of lactic acid attraction. Med Vet Entomol. 1999;13:97-100.

8. Goessens T, Malengier B, Constales D, De Staelen RH. A volume averaging and overlapping domain decomposition technique to model mass transfer in textiles. Accepted in Journal of Computational and Applied Mathematics. 2014; 14 pages. 
9. Santhanam A, Miller MA, Kasting GB. Absorption and evaporation of $\mathrm{n}, \mathrm{n}$-diethyl-m-toluamide from human skin in vitro. Toxicology and Applied Pharmacology. 2005;204(1):81-90.

10. Ye C, Huang H, Fan J, Sun W. Numerical Study of Heat and Moisture Transfer in Textile Materials by a Finite Volume Method. Communications in Computational Physics. 2008;4(4):929-948.

11. Huang HX, Ye CH, Sun W. Moisture transport in fibrous clothing assemblies. Journal of Engineering Mathematics. 2008;61(1):35-54.

12. Tice R, Brevard B. N,N-Diethyl-m-toluamide (DEET), Review of Toxicological Literature. Integrated Laboratory Systems; 1999.

13. Ditzen M, Pellegrino M, Vosshall LB. Insect Odorant Receptors Are Molecular Targets of the Insect Repellent DEET. Science. 2008;319(5871):1838-1842.

14. Syed Z, Leal WS. Mosquitoes smell and avoid the insect repellent DEET. Proceedings of the National Academy of Sciences. 2008.

15. Emission Standards Division. Air emissions models for waste and wastewater EPA-453/R-94-080A. U.S. Environmental Protection Agency; 1994.

16. Hoffmann EJ, Miller JR. Reduction of Mosquito (Diptera: Culicidae) Attacks on a Human Subject by Combination of Wind and Vapor-Phase DEET Repellent. Journal of Medical Entomology. 2002;39(6):935-938.

17. Karr JI, Speaker TJ, Kasting GB. A novel encapsulation of N,N-diethyl-3-methylbenzamide (DEET) favorably modifies skin absorption while maintaining effective evaporation rates. Journal of Controlled Release. 2012;160(3):502 508 .

18. N'Guessan R, Knols BGJ, Pennetier C, Rowland M. DEET microencapsulation: A slow-release formulation enhancing the residual efficacy of bed nets against malaria vectors. Trans R Soc Trop Med Hyg. 2008;102(3):259-262. 
Table 1: Simulation of a bioassay duration test showing how the concentration values in the textile decrease over time

\begin{tabular}{lll}
\hline \hline Duration [h] & Mass DEET $[\boldsymbol{\mu g}]$ & Conc $\left[\mathbf{n m o l} / \mathbf{c m}^{2}\right]$ \\
\hline 0 & 152.5 & 25 \\
1 & 140.5 & 23 \\
3 & 120.5 & 19.8 \\
6 & 97.0 & 15.9 \\
12 & 62.5 & 10.2 \\
24 & 23.2 & 5.3 \\
\hline \hline
\end{tabular}


Table 2: Experimental average DEET concentration values measured with a liquid extraction setup, and the parameters that can be deduced from this.

\begin{tabular}{ccccccc}
\hline \hline Sample & $\begin{array}{c}\text { Avg. start } \\
{\left[\mathrm{nmol} / \mathrm{cm}^{2}\right]}\end{array}$ & $\begin{array}{c}\text { Avg. 91days } \\
{\left[\mathrm{nmol} / \mathrm{cm}^{2}\right]}\end{array}$ & $\begin{array}{c}\text { Diff. } \\
{[\mu \mathrm{g}]}\end{array}$ & $\begin{array}{c}F_{\text {evap }} \\
{\left[10^{-8} \frac{\mu g}{\mathrm{~mm}^{2} \mathrm{~s}}\right]}\end{array}$ & $\begin{array}{c}\tilde{k}_{\text {evap }} \\
{\left[10^{\left.-13 \frac{m m}{\mathrm{~s}}\right]}\right.}\end{array}$ & $\begin{array}{c}h_{\mathrm{lg}} \\
{[\mathrm{mm} / \mathrm{s}]}\end{array}$ \\
\hline A5 & 61.5 & 8 & 23.46 & 1.492 & 5.1 & $0.06410^{-4}$ \\
C6 & 315 & 27.5 & 110.09 & 7.001 & 24 & $0.29910^{-4}$ \\
A3 & 80 & 5 & 28.77 & 1.830 & 6.2 & $0.07710^{-4}$ \\
C3 & 63 & 9 & 20.75 & 1.320 & 4.5 & $0.05610^{-4}$ \\
\hline \hline
\end{tabular}


Table 3: Bed net hut testing, showing the repellency and the feeding inhibition of the products. The number of mosquitoes found in the treatment huts, control hut, and the number of mosquitoes that fed in the control hut is also given

\begin{tabular}{|c|c|c|c|c|c|c|c|}
\hline \multirow[t]{2}{*}{ Product } & \multirow{2}{*}{$\begin{array}{l}\% \\
\text { Repel }\end{array}$} & \multirow{2}{*}{$\begin{array}{l}\text { \% feeding } \\
\text { inhibition }\end{array}$} & \multirow{2}{*}{$\begin{array}{l}\# \text { mosq } \\
\text { test }\end{array}$} & \multirow{2}{*}{$\begin{array}{l}\text { \# mosq. } \\
\text { control }\end{array}$} & \multirow{2}{*}{$\begin{array}{l}\text { \#mosq. } \\
\text { fed } \\
\text { control }\end{array}$} & \multicolumn{2}{|c|}{$\begin{array}{l}\text { \#mosq. \#mosq. } \\
\text { verandaveranda }\end{array}$} \\
\hline & & & & & & test & control \\
\hline$\overline{\text { DEET+ BINDER Y421 }}$ & 100 & 100 & 0 & 16 & 0 & 0 & 7 \\
\hline DEET+ BINDER Y335 & 93 & 100 & 1 & 16 & 0 & 1 & 7 \\
\hline PERM.+DEET Y421 & 100 & 100 & 0 & 22 & 5 & 0 & 8 \\
\hline PERM.+DEET Y335 & 95 & 100 & 1 & 22 & 5 & 1 & 8 \\
\hline PERM.+DEET+BINDER Y421 & 95 & 100 & 1 & 21 & 7 & 1 & 5 \\
\hline PERM.+DEET+BINDER Y335 & 95 & 100 & 1 & 21 & 7 & 1 & 5 \\
\hline DEET Y421 & 98 & 100 & 1 & 43 & 8 & 1 & 15 \\
\hline DEET Y335 & 98 & 100 & 1 & 43 & 8 & 1 & 15 \\
\hline PERM. Y421 & 78 & 100 & 4 & 18 & 7 & 2 & 10 \\
\hline PERM. Y335 & 67 & 100 & 6 & 18 & 7 & 3 & 10 \\
\hline
\end{tabular}


Table 4: Bed net hut retesting after 12 months, showing the repellency and the feeding inhibition of the products. The amount of mosquitoes found in the treatment huts, control hut, and the amount of mosquitoes that fed in the control hut is also given.

\begin{tabular}{|c|c|c|c|c|c|c|c|}
\hline Product & $\begin{array}{l}\% \\
\text { Repel } \\
\end{array}$ & $\begin{array}{l}\% \text { feeding } \\
\text { inhibition }\end{array}$ & $\begin{array}{l}\text { \# mosq. } \\
\text { test }\end{array}$ & $\begin{array}{l}\text { \# mosq. } \\
\text { control }\end{array}$ & $\begin{array}{l}\text { \# mosq. } \\
\text { fed } \\
\text { control }\end{array}$ & $\begin{array}{l}\text { \#mosq. } \\
\text { veranda } \\
\text { test }\end{array}$ & $\begin{array}{l}\text { \#mosq. } \\
\text { veranda } \\
\text { control }\end{array}$ \\
\hline PERM.+DEET Y421 & 94 & 100 & 2 & 32 & 2 & 2 & 15 \\
\hline PERM.+DEET Y335 & 94 & 100 & 2 & 32 & 2 & 1 & 15 \\
\hline DEET Y421 & 98 & 100 & 1 & 66 & 2 & 1 & 46 \\
\hline DEET Y335 & 67 & 100 & 22 & 66 & 2 & 13 & 51 \\
\hline
\end{tabular}




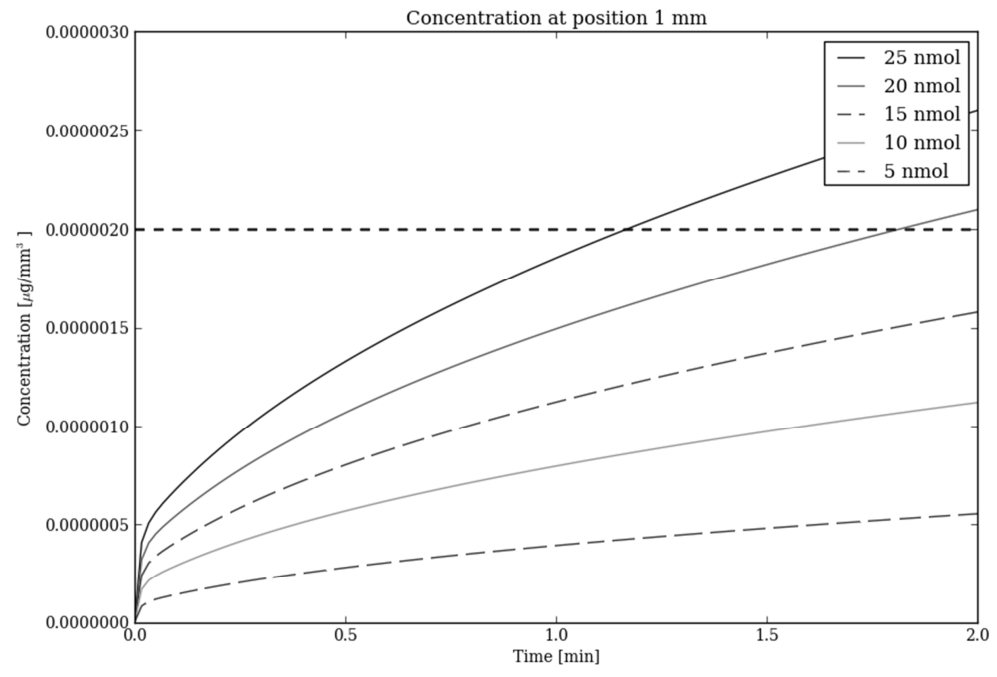

Figure 1: Concentration $1 \mathrm{~mm}$ from the muslin cloth over 2 minutes as obtained with a dose-response simulation. The dashed line is the required amount for good repellency.

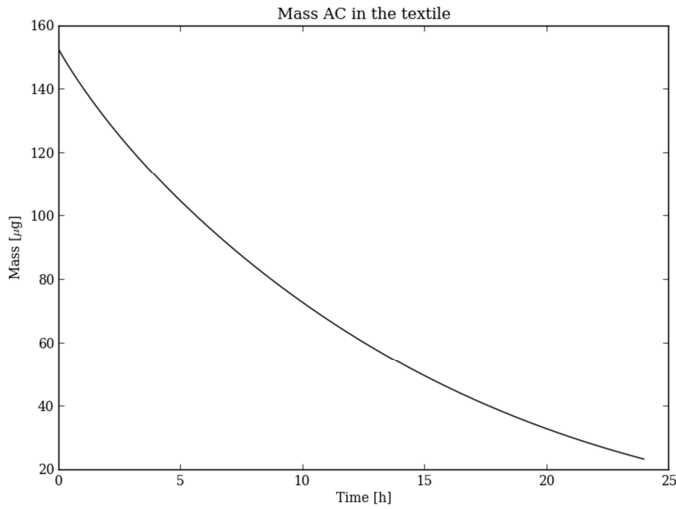

Figure 2: Mass of DEET in the textile, starting with a $25 \mathrm{nmol} / \mathrm{cm}^{2}$ concentration, over 1 day 


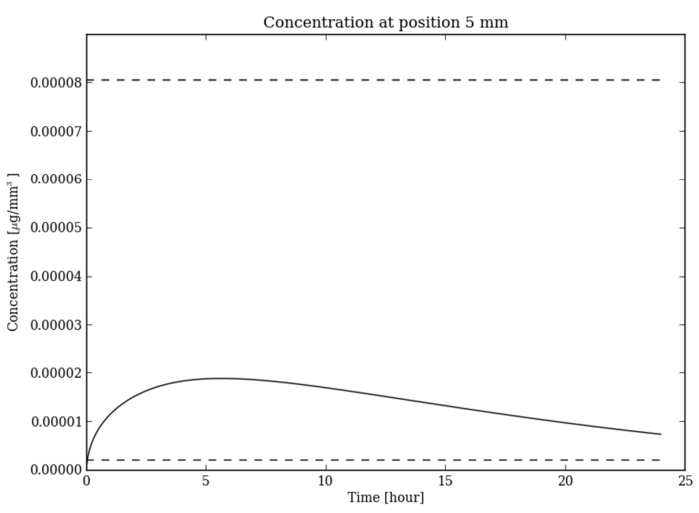

Figure 3: Concentration $5 \mathrm{~mm}$ from the muslin cloth over 1 day as obtained with the simulation code. Top dashed line is the saturation concentration, bottom dash required amount for repellency
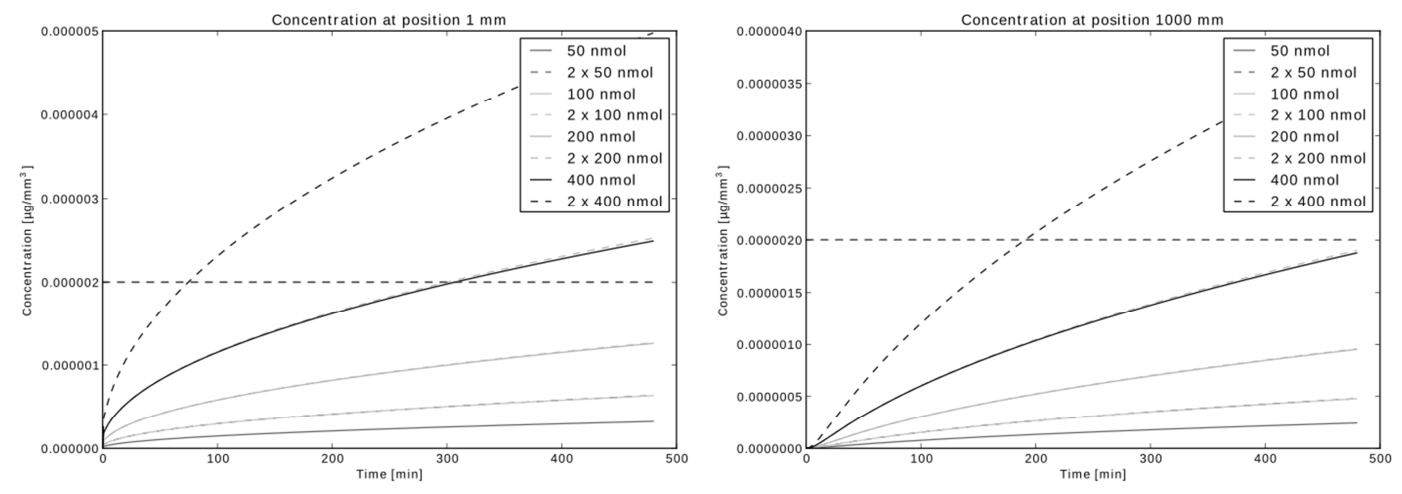

Figure 4: Concentration of DEET due to a single sheet of bed net material, at $1 \mathrm{~mm}$ and $1000 \mathrm{~mm}$ from the curtain. The straight dashed line is the required amount for repellency. The dashed curves are the double of full lines to indicate the effect of a real bed net.

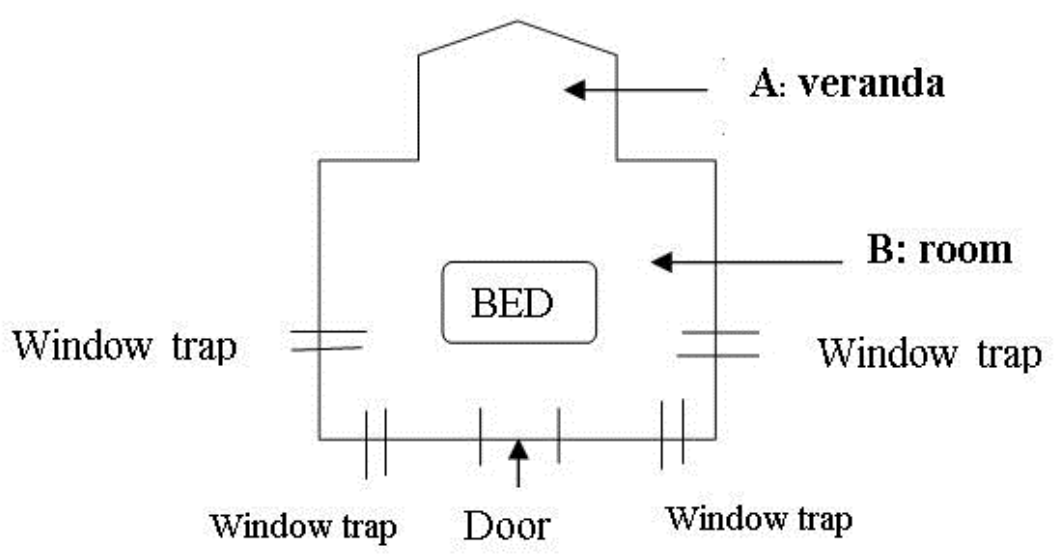

Figure 5: Top view schematic of the sleeping huts. 